\title{
Simulating Quasi-ballistic Transport in Si Nanotransistors
}

\author{
KAUSAR BANOO ${ }^{\mathrm{a}}$, JUNG-HOON RHEW ${ }^{\mathrm{a}}$, MARK LUNDSTROM ${ }^{\mathrm{a}}$, \\ CHI-WANG SHU ${ }^{\mathrm{b}, *}$ and JOSEPH W. JEROME \\ ${ }^{a}$ School of Electrical and Computer Engineering, 1285 EE Building, Purdue University, West Lafayette, IN 47907; \\ ${ }^{\mathrm{b}}$ Div. of Applied Mathematics, Brown University, Providence, RI; \\ ${ }^{\mathrm{c}}$ Dept. of Mathematics, Northwestern University, Evanston, $I L$
}

\begin{abstract}
Electron transport in model $\mathrm{Si}$ nanotransistors is examined by numerical simulation using a hierarchy of simulation methods, from full Boltzmann, to hydrodynamic, energy transport, and drift-diffusion. The on-current of a MOSFET is shown to be limited by transport across a low-field region about one mean-free-path long and located at the beginning of the channel. Commonly used transport models based on simplified solutions of the Boltzmann equation are shown to fail under such conditions. The cause for this failure is related to the neglect of the carriers' drift energy and to the collisiondominated assumptions typically used in the development of simplified transport models.
\end{abstract}

Keywords: MOSFET; Transport; Boltzmann; Ballistic; Hydrodynamic; TCAD

\section{INTRODUCTION}

Nano-scale MOSFETs have low inversion layer mobility and therefore, channel lengths many mean-free-paths long. It has, however, been demonstrated recently that current-day devices operate at roughly $50 \%$ of the ballistic limit [1], and devices that operate at over $70 \%$ have been reported [2]. The reason for this near-ballistic performance is that the critical length that limits current flow is a small portion of the channel near the source [3], so present-day transistors operate in a quasi-ballistic regime. The macroscopic transport models commonly used in TCAD must be questioned in these regimes because they are based on collision-dominated assumptions.

Quasi-ballistic transport has been examined by Baranger and Wilkins with the use of a numerical solution to a simplified Boltzmann equation (onedimensional in velocity and position space and a relaxation time approximation to the scattering integral) [4]. Others have used a similar kinetic equation as a benchmark for examining the performance of macroscopic transport models in the quasi-ballistic regime (e.g. [5]). In this paper, we: (i) explain why present-day MOSFETs with

*Corresponding author. Tel.: (765) 494-3515, Fax: (765) 494-6441, e-mail: lundstro@purdue.edu 
channel lengths that are still several mean-freepaths in length operate surprisingly close to their ballistic limit, (ii) examine quasi-ballistic transport in a model nanoscale transistor $\left(a \mathrm{n}^{+} \mathrm{pn}^{+}\right.$diode) using as a computational benchmark a numerical solution to the full Boltzmann equation (i.e., threedimensional in velocity space with a realistic collision integral appropriate for electrons in silicon), (iii) critically assess common transport models against this computational benchmark, and show that they fail in the quasi-ballistic regime where transistors now operate, and (iv) explain the underlying reasons for this failure. Much of the previous work on ballistic transport has examined conduction in GaAs with electron mobilities on the order of $10,000 \mathrm{~cm}^{2} / \mathrm{V}$-s and even higher (e.g. $[4,5])$. In this paper, we explain why presentday silicon transistors with mobilities 100 times lower still operate in a quasi-ballistic regime. We conclude that low order moment-type transport models cannot work in the quasiballistic regime. This conclusion agrees with that of Nekovee $e t$ al. [6] who examined transport in a much higher mobility GaAs n-i-n diode with a relaxation time approximation that is expected to exaggerate ballistic effects [4]. On the basis of our work on silicon transistors with a realistic treatment of scattering and previous work on compound semiconductors, it now seems clear that a new class of transport models that work in both the ballistic and collision-dominated regimes must be developed.

Before proceeding, we first describe the essential physical picture of carrier transport in a nanoscale MOSFET under high drain bias. As shown in Figure 1, thermal equilibrium carriers are injected from the source across a barrier (whose height is controlled by the gate voltage) into the channel of the MOSFET. The carrier density at the beginning

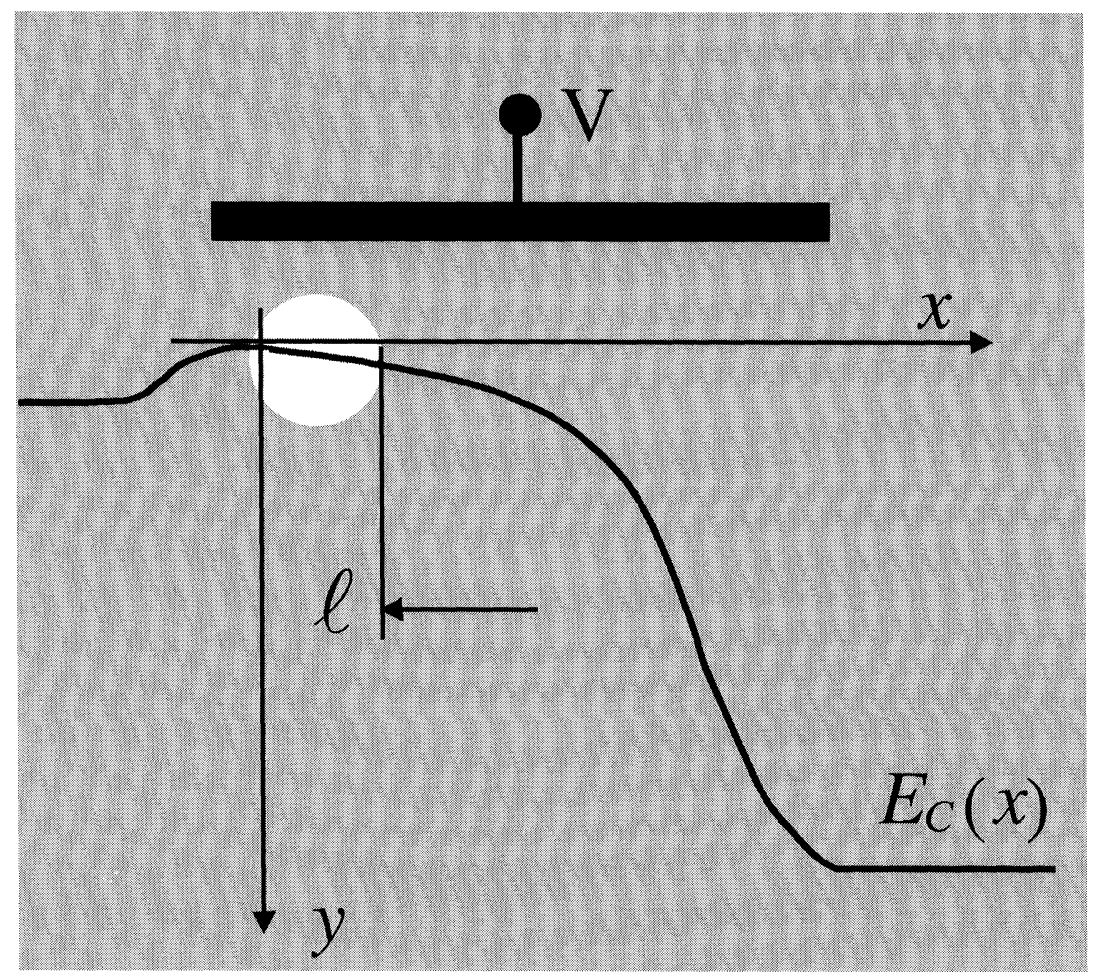

FIGURE 1 Sketch of the depth-averaged conduction band profile $v s$. position along the channel for a MOSFET at high drain-tosource and gate-to-source bias. 
of the channel is set at a nearly constant value by MOS electrostatics. Carriers traverse a low-field region of length, $\ell$, then are collected by the highfield portion of the channel. Off-equilibrium transport in the high-field portion of the channel produces strong velocity overshoot. When overshoot is strong, the high-field region acts as a nearperfect absorber for electrons, and very few back scatter into the low-field region. The fraction of electrons injected into the low-field region that backscatter is related to the length of the region and the mean-free-path by [3]

$$
r=\frac{\ell}{\ell+\lambda}
$$

where $\lambda$ is the mean-free-path for backscattering. For current technology, $r \approx 0.5$, which implies that the length of the critical region is about one meanfree-path. Transport in a MOSFET under on-current conditions, therefore, involves the transport of near-equilibrium carriers across a region about one mean-free-path long followed by off-equilibrium transport in the short, high-field part of the channel. Since velocity overshoot in short high-field regions is now well understood [7,8], we concentrate on the quasi-ballistic transport of near-equilibrium carriers across the short, lowfield region.

\section{TRANSPORT IN A MODEL TRANSISTOR}

Figure 2 shows a very simple, model transistor that we use to examine quasi-ballistic transport across a thin, zero-field region. The carrier concentration at $x=0$ is fixed to represent the effect of MOS electrostatics which holds the inversion layer density nearly constant at the beginning of the channel. The length of the zero-field region is set at $20 \mathrm{~nm}$, which corresponds to about one-mean-free path for backscattering (at the assumed inversion layer mobility of $450 \mathrm{~cm}^{2} / \mathrm{V}$-s). The electric field in the high-field region is assumed to be constant and increases in magnitude to represent the effect of an

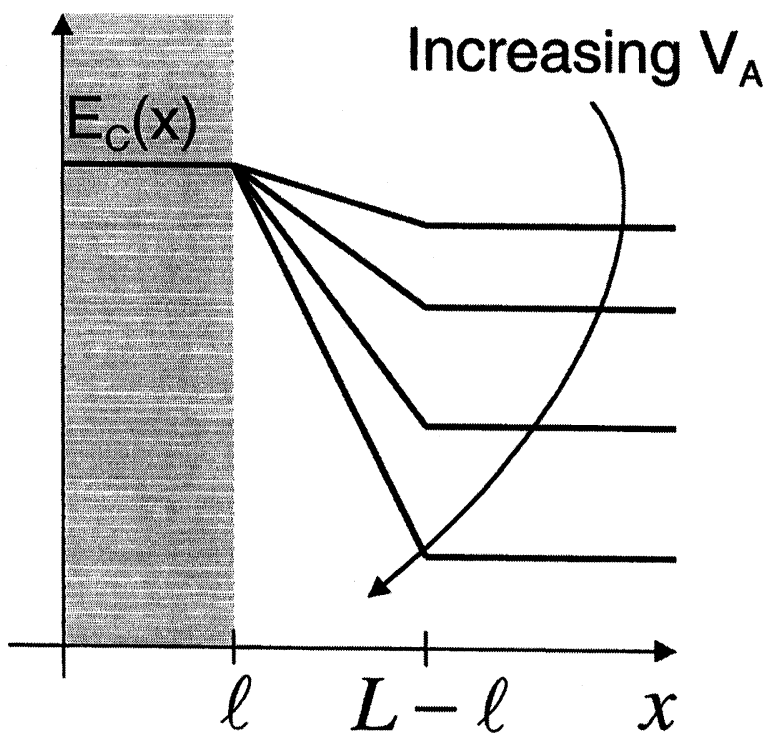

FIGURE 2 Sketch of a simple, model transistor for exploring non self-consistent transport under conditions representative of a MOSFET at high drain-to-source and gate-to-source bias.

increasing drain bias. Steady-state electron transport was modeled by solving the Boltzmann Transport Equation (BTE) using a scattering matrix approach $[9,10]$ with standard energy band and scattering models for electron transport in $\mathrm{Si}$ [11]. We also examined transport using driftdiffusion and energy transport models calibrated to the same physical model used in the solution of the full BTE. The results of these simulations are described in detail in [12].

In the limit of high electric fields, the high-field region acts as a perfect absorber to the low-field region. Under such conditions, we do not expect the diffusion velocity to exceed the thermal velocity. (Actually, since carriers directed normal to the zero-field slab have a higher probability of transmitting, the velocity at the absorbing contact can exceed the thermal velocity of a hemiMaxwellian by as much as $\approx 20 \%$ in the case of anisotropic scattering such as ionized impurity scattering [13]). Figure 3 shows the average velocity at the end of the low-field region, as a function of the applied bias. Also shown is the perfect absorber limit computed by replacing the high-field region 


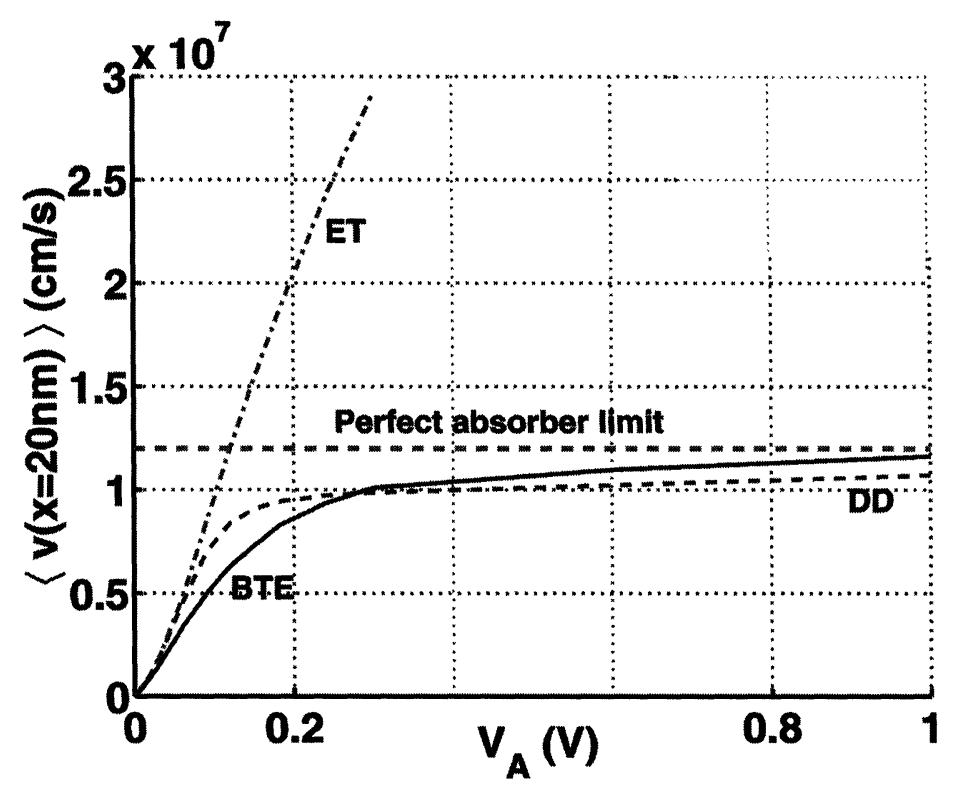

FIGURE 3 Average velocity at $\mathrm{x}=20 \mathrm{~nm}$ (the end of the zero-field region) $v s$. applied bias (electric field times the length of the high field region). The dashed line is the perfect absorber limit.

with a perfectly absorbing contact. Note that the velocity from the BTE solution saturates at the perfect absorber limit as the applied bias increases. The velocity from the drift-diffusion simulation saturates at $1 \times 10^{7} \mathrm{~cm} / \mathrm{s}$ and does not display the velocity overshoot that must occur in the short, high-field region (which is not shown in Fig. 3). However, because the thermal velocity in $\mathrm{Si}$ happens to be numerically almost identical to the saturated velocity, the DD simulation produces approximately the correct velocity at the beginning of the high-field region, but if the simulation had been done self-consistently, the wrong electric field profile would have resulted.

The energy transport simulation captures the velocity overshoot that occurs in the high-field region, but it does not limit the velocity in the lowfield region to the thermal velocity as it should. As the bias increases, the velocity in the zero field region increases without bound; carriers to diffuse faster than the thermal velocity across the zero field region, which is non-physical. Failure to properly describe transport across such a region has important consequences for nanoscale
MOSFETs, because transport across the low-field region at the beginning of the channel is the current-limiting process.

\section{TRANSPORT IN AN n-i-n DIODE}

To capture self-consistent effects, the most popular model device to represent a transistor is the $\mathrm{n}^{+} \mathrm{pn}^{+}$diode. We used a device with a $50 \mathrm{~nm}$ long lightly doped, p-type region to represent a MOSFET with a $50 \mathrm{~nm}$ long-channel. Four consistent transport models were used to study this device - the Boltzmann Transport Equation (BTE) and three of its macroscopic approximations. The BTE assumes a simple, spherical nonparabolic energy band with acoustic and optical (intervalley) phonon scattering with parameters calibrated to reproduce the velocity-field and energy-field characteristics of bulk Si [11]. It is solved by directly discretising the distribution function on a grid in position and momentum space, making no approximations about the shape. This direct solution is essentially a scattering 
matrix solution which does not make use of precomputed scattering matrices so that position and momentum space grids can be easily refined (details of the numerical techniques will be discussed elsewhere [14]). The three macroscopic transport models used for comparison are driftdiffusion (DD), hydrodynamic (HD), and energy transport (ET) [15]. The essential difference between the hydrodynamic and energy transport models is the neglect of the drift energy in the energy transport equation. Their parameters are also calibrated to bulk $\mathrm{Si}$ to ensure that all the transport models represent a different approximation to the same physical problem. The hydrodynamic and energy transport models were solved by the essentially nonoscillatory (ENO) method [16]. All transport models were solved selfconsistently with Poisson's equation.

BTE simulations were conducted with both realistic Si scattering rates and with zero scattering (to establish the ballistic limit). The computed I-V characteristics, shown in Figure 4, show that the device operates within about $50 \%$ of its ballistic limit, roughly the same as a present-day MOSFET. The DD results show highly non-physical behavior within the device, but the $I-V$ characteristic is remarkably close to the BTE. Both the HD and ET models produce currents that are higher that the BTE simulation. The ET simulation actually gives a current that is above the ballistic limit.

Figure 5 is a plot of the average velocity $v s$. position at the highest applied bias. The driftdiffusion velocity, which is clamped at the saturation velocity, is highly non-physical. The hydrodynamic model produces much higher velocities than the BTE from which it was derived, and the energy transport model produces velocities that are still higher-higher, in fact, over most of the channel region, than the ballistic velocity. As shown in Figure 6, both the HD and ET models do a reasonable job with the carrier temperature (energy).

There are, of course many different ways to formulate macroscopic transport models depending on how one truncates the hierarchy, and the various ways to formulate relaxation times, heat flux, etc. $[17,18]$. The results shown in Figures 5 and 6 demonstrate that at least one typical formulation of the equations produces serious

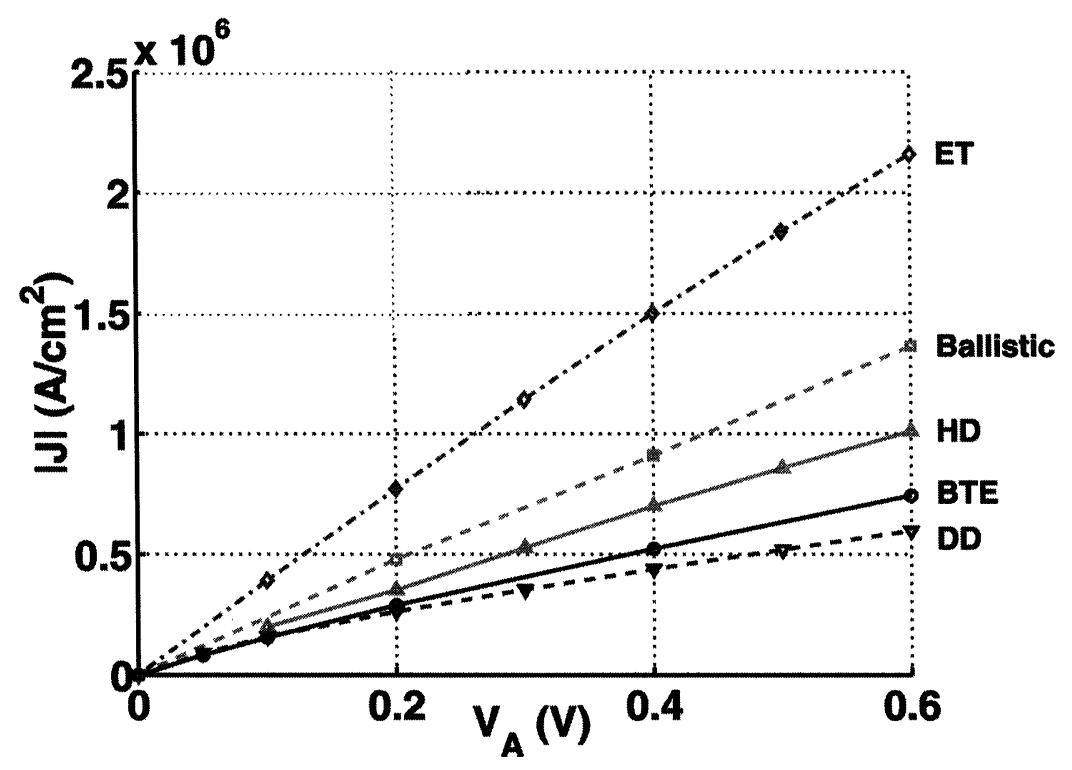

FIGURE 4 The computed current $v s$. voltage characteristic of the $\mathrm{n}^{+}-\mathrm{p}-\mathrm{n}^{+}$diode. 


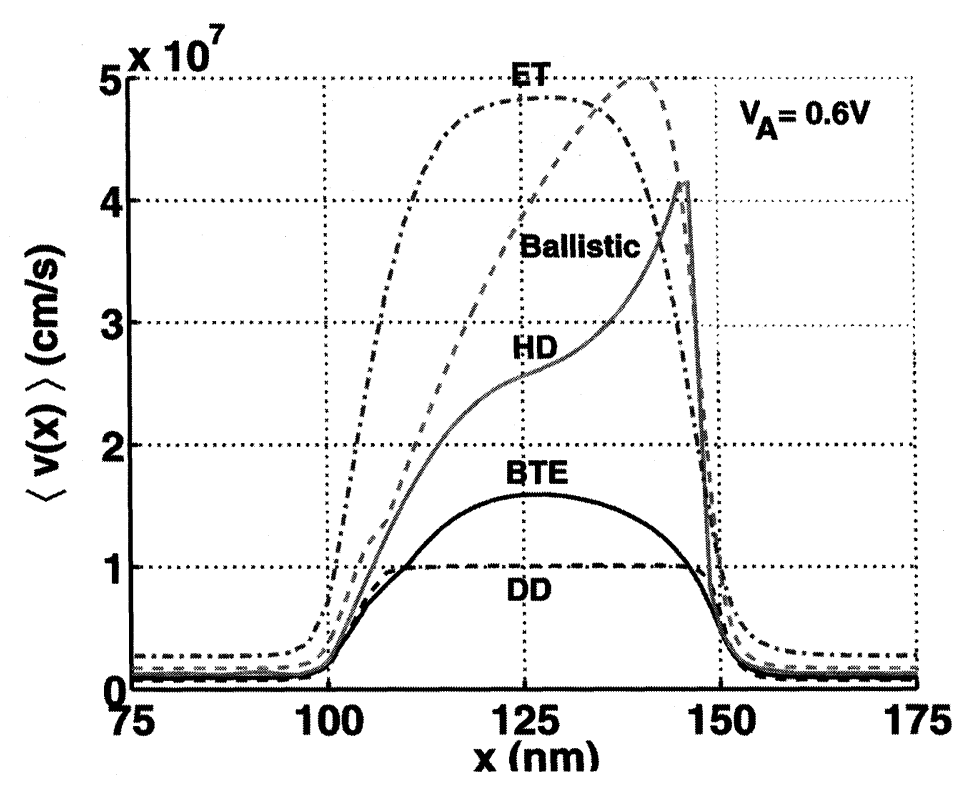

FIGURE 5 The average velocity $v$ s. position in the $\mathrm{n}^{+}-\mathrm{p}-\mathrm{n}^{+}$diode at an applied bias of $0.6 \mathrm{~V}$.

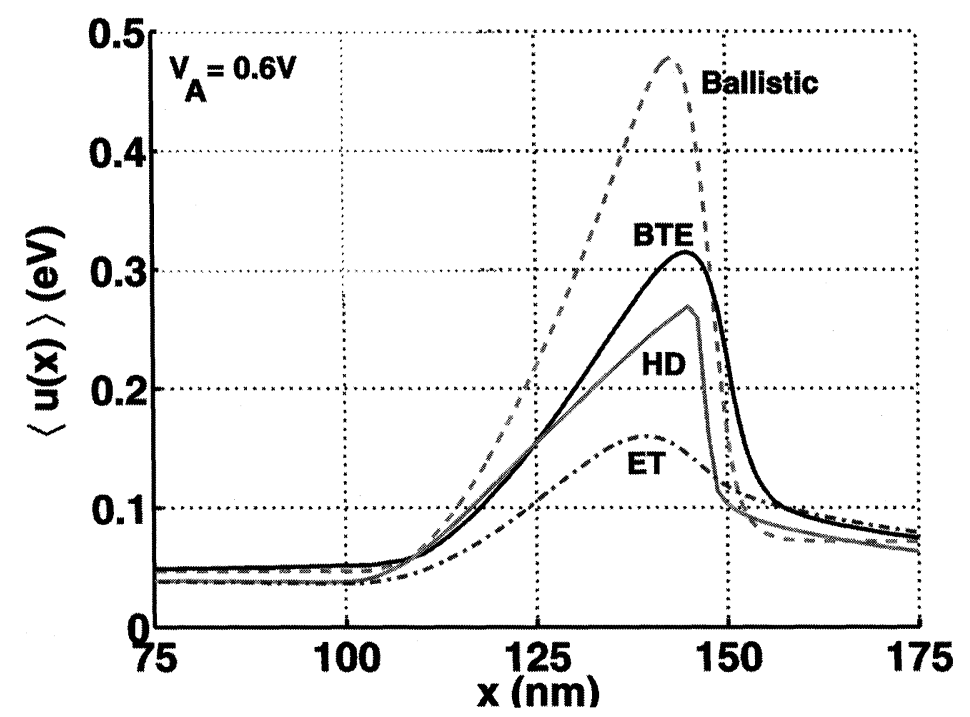

FIGURE 6 The average kinetic energy vs. position in the $\mathrm{n}^{+}-\mathrm{p}-\mathrm{n}^{+}$diode at an applied bias of $0.6 \mathrm{~V}$.

errors for nanoscale transistors. Figure 7 shows why we believe that this failure cannot be avoided in HD and ET type of transport models, irrespective of how they are formulated. Figure 7 plots the ratio of the negatively directed flux to the positively directed flux as obtained from the BTE solution (this ratio is a measure of the anisotropy in the carrier distribution). Note that the minimum in this ratio occurs at the top of the potential barrier, which is the beginning of the low field portion of the channel and that its value is only 0.16 . For this bias, the carriers are primarily 


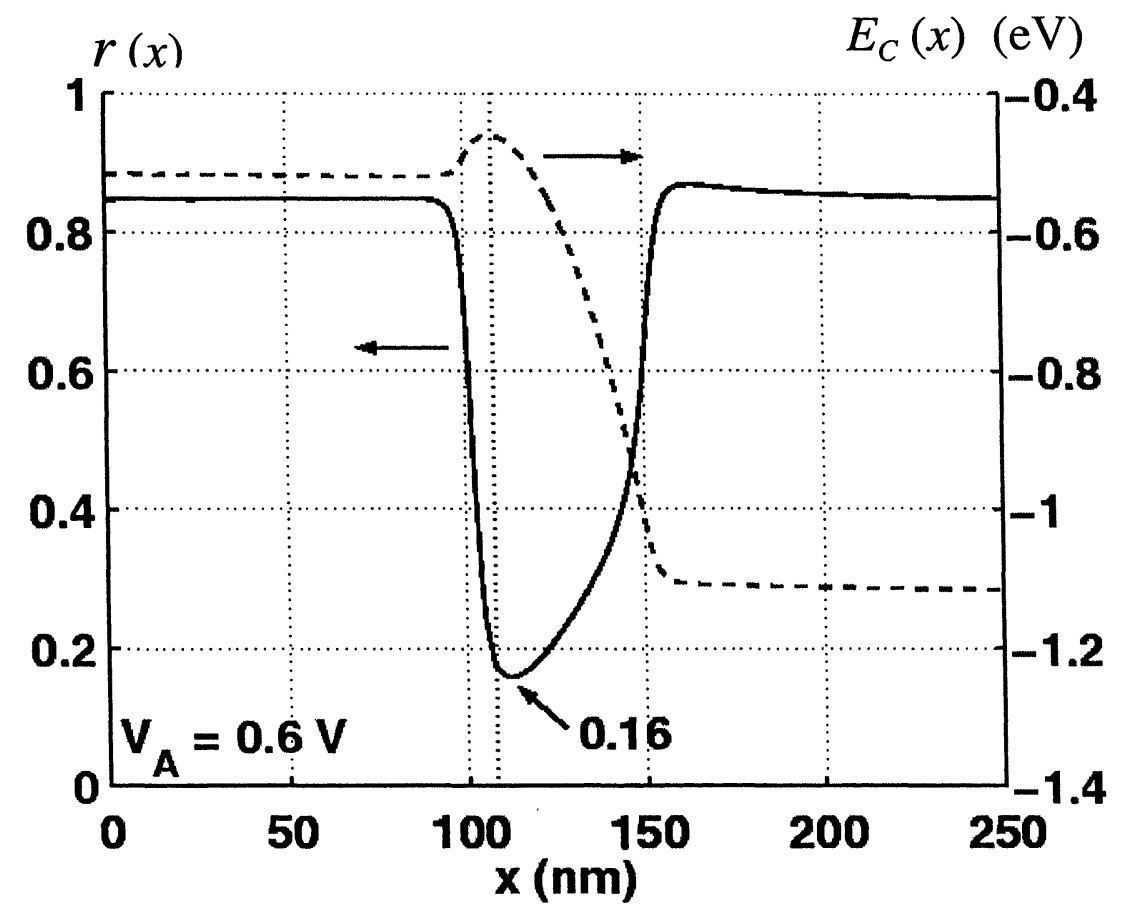

FIGURE 7 The ratio, $\mathrm{J}^{-} / \mathrm{J}^{+} v$ s. position for the $\mathrm{n}^{+}-\mathrm{p}-\mathrm{n}^{+}$diode at an applied bias of $0.6 \mathrm{~V}$. This ratio is a measure of anisotropy in the carrier velocity distribution and is zero under ballistic conditions. Also shown is the conduction band edge vs. position.

injected from the source, and the small ratio indicates that little backscattering occurs in the low-field region. Transport is nearly ballistic and the distribution function is highly anisotropic. Since HD/ET types of transport models usually make use of parameters from bulk transport, where the distribution is nearly isotropic, they are unlikely to be able to describe transport in the ballistic limit.

\section{DISCUSSION}

The causes of the failure of common macroscopic transport models in the near-ballistic regime can be traced to the assumption used in their derivation. For example, the kinetic energy of carriers can be written as

$$
u=\frac{3}{2} k_{B} T_{e}+\frac{1}{2} m^{*}\langle\nu\rangle^{2}
$$

where the first term is the thermal energy that arises from scattering and the second is the drift energy associated with the average motion of the ensemble. It is common to ignore the second term, but in the ballistic limit, there is no scattering to increase the temperature, and the second term dominates. Indeed, Baccarani and Wordemann have shown that the drift energy is responsible for the saturation of the diffusion velocity to the thermal velocity in steep gradients of carrier concentration [19]. The neglect of this term in the energy transport model is a likely cause of the unphysically high diffusion velocities observed in the HD/ET simulations reported here.

Another cause for the failure of macroscopic transport models lies in the use of diffusive assumptions in their derivation. For example, relaxation times are usually taken from bulk simulations where the distribution is nearly isotropic; they cannot be applied to quasi-ballistic devices where the carrier distribution is highly 
anisotropic without producing the types of errors reported here.

The formulation of a computationally efficient transport model that works from the diffusive to the ballistic regimes is a key challenge for the device modeling and simulation community. One possibility is the use of flux methods (e.g. [20]) which describe portions of the distribution, rather than the entire distribution. As a result, highly anisotropic distributions can be treated. A tractable, off-equilibrium version of the flux equations has not yet, however, been reported. Another issue is the choice of a model device for examining transport approaches. Two-dimensional electrostatics is a critical feature of the MOSFET that is not at all captured in the $n^{+}-p-n^{+}$device. Full 2D transport, however, greatly complicates the problem and makes it difficult to rapidly assess a variety of transport models. In a MOSFET, however, inversion layer transport dominates and is predominantly one-dimensional. A numerical charge sheet model, which treats electrostatics two-dimensionally and transport one-dimensionally, should therefore, be a realistic model for a MOSFET as well as provide a simple means to explore transport models.

\section{SUMMARY}

The results of this study show that transport models currently used in TCAD do not describe transport in the ballistic regime and produce serious errors in the quasi-ballistic regime where devices now operate $[1,2]$. The reasons for this failure were explained in terms of the assumptions made when deriving them from the Boltzmann equation. A new approach for developing macroscopic transport models, valid in both the collision-dominated and collision-free regimes, needs to be developed. This work is important to ensure that semiclassical transport is modeled correctly in nanotransistors and also to ensure that quantum hydrodynamic transport models accurately capture both classical and quantum mechanical effects.

\section{Acknowledgements}

This work was supported by the following agencies: NSF Distributed Center for Advanced Electronic Simulation (DesCArtES), the Semiconductor Research Corporation, NSF grant DMS9704458,NSF grant ECS-9906606 and ARO grant DAAG55-97-1-0318.

\section{References}

[1] Assad, F., Ren, Z., Bendix, P. and Lundstrom, M. S., "Performance Limits of Si MOSFET's", IEDM Tech. Digest., p. 547, Dec. 1999.

[2] Timp, G., Bude, J. et al., "The Ballistic Nanotransistor", IEDM Tech. Digest., p. 55, Dec. 1999.

[3] Lundstrom, M. S. (1997). "Elementary scattering theory of the MOSFET", IEEE Electron Dev. Lett., 18, 361-363.

[4] Baranger, H. U. and Wilkins, J. W. (1987). "Ballistic structure in the electron distribution function of small semiconducting structure: General features and specific trends", Phys. Rev. B, 36, 1487-1502.

[5] Cerignani, C., Gamba, I. M., Jerome, J. W. and Shu, C.-W. (2000). "Device benchmark comparisons vias kinetic, hydrodynamic, and high-yield models", Comput. Methods Appl. Mech. Engrg., 181, 381-392.

[6] Nekovee, M., Geurts, B. J., Boots, H. M. J. and Schuurmans, M. F. H. (1992). "Failure of extendedmoment-equation approaches to describe ballistic transport in submicrometer structures", Phys. Rev. B, 45, 6643-6651.

[7] Pinto, M. R., Sangiorgi, E. and Bude, J. (1993). "Silicon transconductance scaling in the overshoot regime", IEEE Electron Dev. Lett., 14, 375-378.

[8] Laux, S. E. and Fischetti, M. V. (1988). "Monte Carlo simulation of submicrometer Si n-MOSFET's at 77 and 300 K", IEEE Electron Dev. Lett., 9, 467-469.

[9] Das, A. and Lundstrom, M. S. (1990). "A scattering matrix approach to device simulation", Electron., 33, 1299- 1307 .

[10] Stettler, M. A. and Lundstrom, M. S. (1992). "Selfconsistent scattering matrix calculation of the distribution function in semiconductor devices", Appl. Phys. Lett., 60, 2908-2910.

[11] Jacoboni, C. and Reggiani, L. (1983). "The Monte Carlo method for the solution of charge transport in semiconductors with applications to covalent materials", Reviews of Modern Physics, 55, 645.

[12] Kausar Banoo and Mark Lundstrom, "Electron Transport in a Model Si Transistor", to appear in Solid-state Electron., 2000.

[13] Berz, F. (1974). "Diffusion near an absorbing boundary", Electron., 15, 1245-1255. 
[14] Banoo, K., Lundstrom, M. S. and Smith, R. K., "Direct Solution of the Boltzmann Transport Equation in Nanoscale Si Devices", to be presented at SISPAD 2000, Seattle, W. A. September 2000.

[15] Jerome, J. W. and Shu, C.-W. (1994). Semiconductors, Part II, p.185, Springer-Verlag.

[16] Shu, C.-W. and Osher, S. J. (1988). J. Comp. Phys., 77, 439.

[17] Mark Lundstrom (1990). Fundamentals Carrier Transport, Addison-Wesley, Reading, MA.
[18] Stettler, M. A., Alam, M. A. and Lundstrom, M. S. (1993). "A Critical Examination of the Assumptions Underlying Macroscopic Transport Equations for Silicon Devices", IEEE Trans. Electron Dev., 40, 733-740.

[19] Baccarani, G. and Wordemann, M. R. (1985). "An Investigation of Steady-State Velocity Overshoot in Silicon", Electron., 28, 407-416.

[20] Assad,F., Banoo, K. and Lundstrom, M.(1998). "The DriftDiffusion Equation Revisited”, Electron., 42, 283-295. 

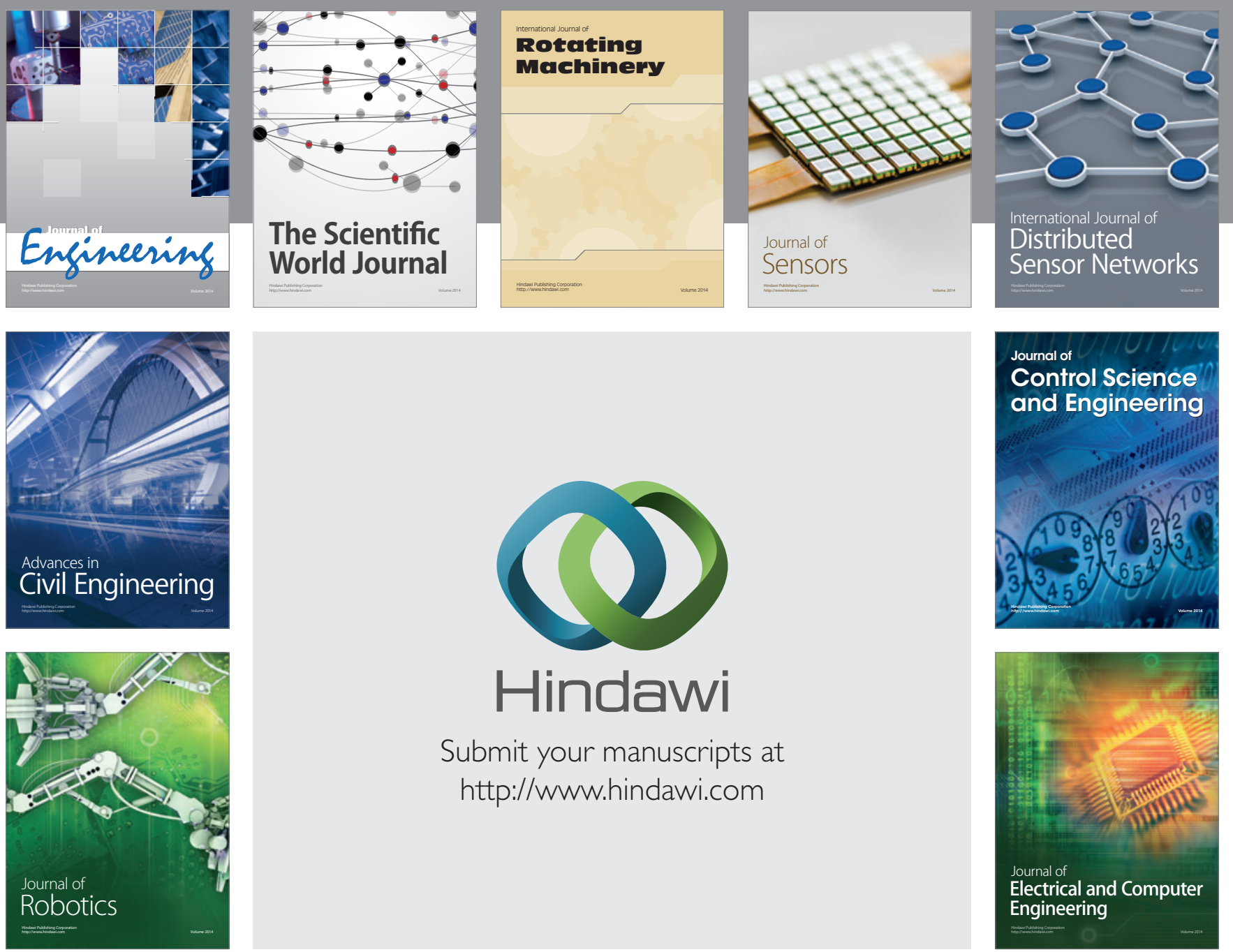

Submit your manuscripts at

http://www.hindawi.com
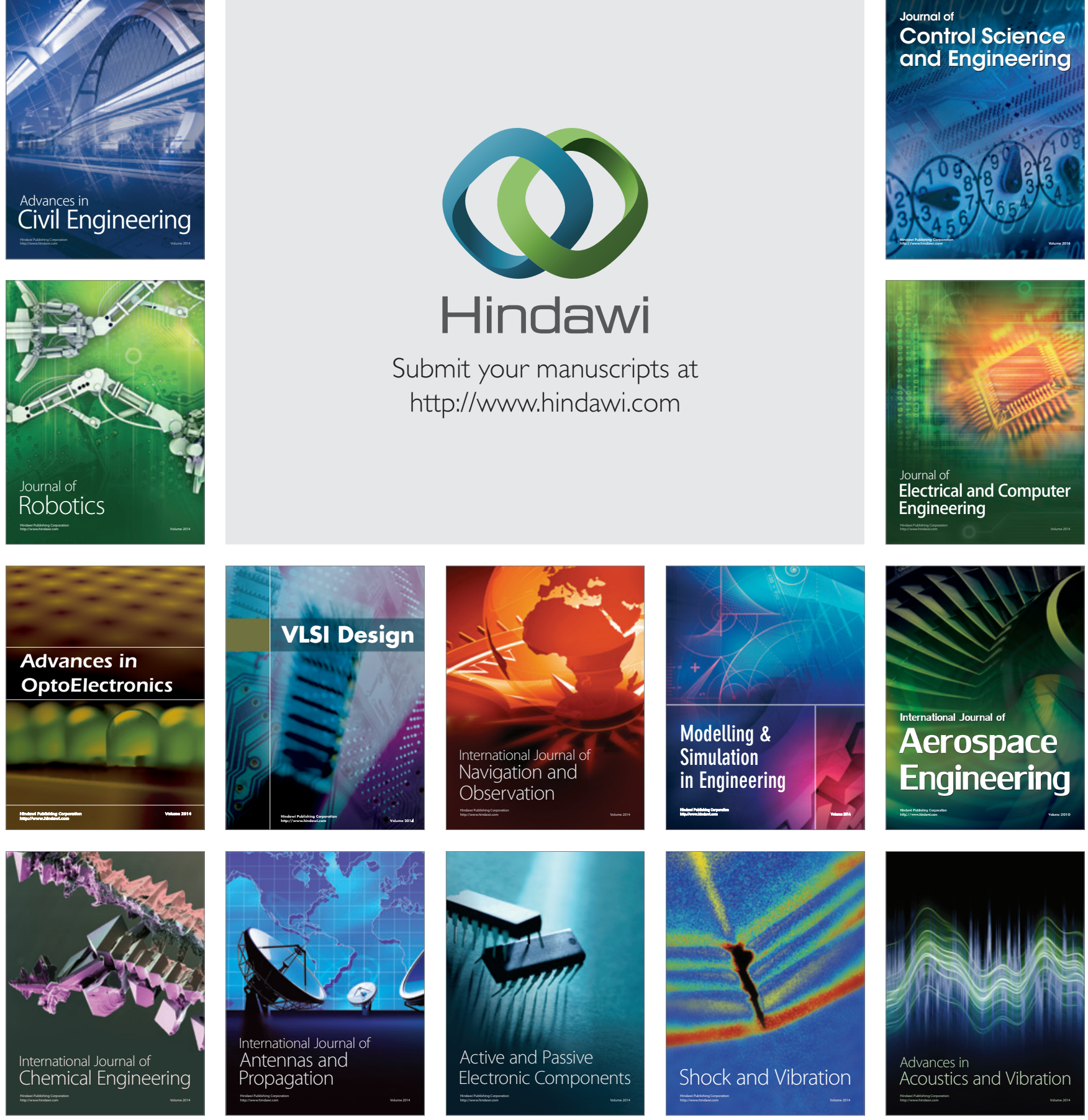